\title{
Evaluating neighborhood structures for modeling intercity diffusion of large-scale dengue epidemics
}

\author{
Tzai-Hung Wen ${ }^{1 *}$, Ching-Shun Hsu and Ming-Che Hu²
}

\begin{abstract}
Background: Dengue fever is a vector-borne infectious disease that is transmitted by contact between vector mosquitoes and susceptible hosts. The literature has addressed the issue on quantifying the effect of individual mobility on dengue transmission. However, there are methodological concerns in the spatial regression model configuration for examining the effect of intercity-scale human mobility on dengue diffusion. The purposes of the study are to investigate the influence of neighborhood structures on intercity epidemic progression from pre-epidemic to epidemic periods and to compare definitions of different neighborhood structures for interpreting the spread of dengue epidemics.
\end{abstract}

Methods: We proposed a framework for assessing the effect of model configurations on dengue incidence in 2014 and 2015, which were the most severe outbreaks in 70 years in Taiwan. Compared with the conventional model configuration in spatial regression analysis, our proposed model used a radiation model, which reflects population flow between townships, as a spatial weight to capture the structure of human mobility.

Results: The results of our model demonstrate better model fitting performance, indicating that the structure of human mobility has better explanatory power in dengue diffusion than the geometric structure of administration boundaries and geographic distance between centroids of cities. We also identified spatial-temporal hierarchy of dengue diffusion: dengue incidence would be influenced by its immediate neighboring townships during pre-epidemic and epidemic periods, and also with more distant neighbors (based on mobility) in pre-epidemic periods.

Conclusions: Our findings suggest that the structure of population mobility could more reasonably capture urbanto-urban interactions, which implies that the hub cities could be a "bridge" for large-scale transmission and make townships that immediately connect to hub cities more vulnerable to dengue epidemics.

Keywords: Dengue, Epidemic diffusion, Spatial regression, Human mobility, Taiwan

\section{Background}

Dengue fever is a vector-borne infectious disease that is transmitted by contact between vector mosquitoes and susceptible hosts [1]. Since the 1970s, dengue fever has been gradually spreading throughout tropical and subtropical countries, and its transmission involves interactions among carriers, mosquitoes, and healthy humans. More than 125 countries are impacted by the disease, and

\footnotetext{
*Correspondence: wenthung@ntu.edu.tw

1 Department of Geography, National Taiwan University, No. 1, Sec. 4,

Roosevelt Road, Taipei City 10617, Taiwan

Full list of author information is available at the end of the article
}

it is an increasingly serious threat to global public health due to climate change. Previous studies showed meteorological and social-economic risk factors that facilitate the disease transmission, including temperature, rainfall, population density, demographic composition, urbanized levels and more [2-4]. The Fifth Assessment Report (AR5) of the Intergovernmental Panel on Climate Change (IPCC) also confirmed that global warming would create more suitable habitats for vector mosquitoes in sub-tropical regions and speed up the geographic expansion of dengue epidemic areas due to global mobility including

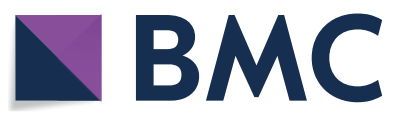

(c) The Author(s) 2018. This article is distributed under the terms of the Creative Commons Attribution 4.0 International License (http://creativecommons.org/licenses/by/4.0/), which permits unrestricted use, distribution, and reproduction in any medium, provided you give appropriate credit to the original author(s) and the source, provide a link to the Creative Commons license, and indicate if changes were made. The Creative Commons Public Domain Dedication waiver (http://creativecommons.org/ publicdomain/zero/1.0/) applies to the data made available in this article, unless otherwise stated. 
to some high-latitude countries such as France and Japan [5-12].

Due to the limited flight range of mosquitoes [13], it is impossible for the virus to be transmitted to distant areas by dengue vectors. Population movement across countries by air traffic is the major driver of the international spread of the disease [12-17]. Via air travel, disease importation from dengue-endemic countries is a trigger point for initiating indigenous epidemics in some dengue-epidemic countries or regions, such as Tokyo, Japan; south-east France; and southern Taiwan [14, 18]. Routine mobility behaviors, such as daily commutes, are also drivers of large-scale intercity transmission [19]. Therefore, understanding the spatial structure of population mobility is crucial for assessing the possible mechanisms of dengue diffusion and identifying the geographic characteristics of high-risk areas [20].

Recent studies on assessing the influence of human mobility on dengue transmission can be categorized into three approaches. The first is to construct simulation or statistical models that incorporate human mobility as the mechanism of dengue diffusion [21]. For example, Barmak et al. [22] showed that the long-distance mobility pattern is an efficient pathway for dengue transmission. Another study used survival analysis to show that daily routine commuters facilitate the large-scale spatial-temporal diffusion of the epidemic in a city [19]. The second perspective is to collect human mobility or behavior data to analyze the spread of dengue. Stoddard et al. [15] showed that small-scale mobility behavior among households also played an important role in dengue epidemics in Iquitos, Peru. Wesolowski et al. [16] used the Call Detail Records (CDRs) from mobile phones to analyze the spatial behaviors of humans in Pakistan for predicting diffusion of dengue in time and space. Airline traffic data are also available for studying the international spread of dengue epidemics and assessing the disease importation risk from dengue-endemic countries [23, 24]. The third perspective is to analyze geometric structures of geography to measure geospatial similarity or neighborhoods as a surrogate for human mobility. Spatial regression modeling is the major approach for measuring the neighborhood effects on dengue risk after controlling for environmental factors $[4,25]$.

The above studies showed that human mobility could be the main risk factor for dengue transmission on both the regional and global scales. However, methodological concerns remain for examining the effect of intercity human mobility. First, spatial settings in the regression model often examine the geometric relationships of geography as a surrogate for spatial interactions and human interactions. For example, the weights of spatial contiguity can be defined as administration boundaries with common borders and points [26] or areas based on $\mathrm{k}$-nearest neighbors within a specific distance [25]. These definitions may simplify the complex interactions of humans because the geometry of spatial contiguity cannot comprehensively reflect these human interactions due to topographical or social-economic barriers across the study area [27]. Moreover, the spatial heterogeneity of human interactions or mobility may not be captured by the geometry of the boundaries alone. In addition, it is difficult to differentiate the effect of urban-to-urban or rural-to-rural mobility on epidemic diffusion if these areas share similar geometric structures. Some studies suggest that the use of real population flow could act as a spatial weight that captures more realistic spatial interactions [27, 28]. Therefore, approaches that use Global Positioning System (GPS) logs, cell phone records or geotags from social media for tracking moving trajectories of individuals have become emerging methods for studying human mobility and dengue risk [29, 30]. However, massive cell phone data provided by telecommunication companies are often difficult to access in the research community. Tracking collective behaviors from cell phone data may also violate location privacy, and this approach could be controversial in most developed countries. Due to these concerns, mathematical models, such as gravity, spatial interaction or radiation models, are used to estimate population flow across cities. Spatial models have become widely used approaches to study the geography of human mobility and disease transmission $[29,31,32]$. Among these models, the radiation model is a parameter-free algorithm that is robust in estimating the flow of intercity human mobility [32].

To clarify the role of intercity human mobility, we used the radiation model to capture the structure of spatial mobility as a possible mechanism. We examined the effect of neighborhood structures on the spatial-temporal spread of dengue epidemics in southern Taiwan from 2014 to 2015, the most severe outbreaks over the course of 70 years in Taiwan, and identified the common socialdemographic features in these high-epidemic regions. By profiling the neighborhood effects on the spatial-temporal structures of disease spread, we proposed a study framework for interpreting possible pathways of intercity diffusion of dengue epidemics. The purposes of the study are (1) to investigate the influence of neighborhood structures on epidemic progression from the pre-epidemic to epidemic periods and (2) to compare the definitions of different neighborhood structures for interpreting the spread of dengue epidemics. 


\section{Data and methods}

\section{Study area}

Southeast Asia is one of the major dengue-endemic regions in the world [33-36]. Taiwan is located in the border region of Southeast and East Asia. Southern Taiwan, which is passed through by the Tropic of Cancer, has a tropical monsoon climate; it is dry in the winter and hot and wet in the summer and autumn. The population has grown quickly, reaching 5.5 million in 2014 . With an average of 683 persons $/ \mathrm{km}^{2}$, metropolitan areas of southern Taiwan have become one of the most densely populated areas in the world. Due to its climatic and demographic characteristics, the region is a severe dengue-epidemic region of Taiwan, which annually covers more than $85 \%$ of the total confirmed dengue cases in Taiwan. Therefore, this region, including Tainan and Kaohsiung Cities and Pin-tung County, was used as the study area. A township was used as the unit for analysis, which is the basic unit for regional master planning and national policy implementation. Our study analyzed the dengue incidence and profiled the social-economic structures of spatial diffusion in 108 townships from 2014 to 2015. To differentiate between the social-economic statuses of each township, we categorized the urbanization levels into seven types, including highly or middle-developed, emerging, general, aging, rural, and non-developed areas as shown in Fig. 1. These types were determined by sociodemographic variables, including population density, population ratio of people with college or above educational levels, population ratio of elder people over 65 years old, population ratio of people of agriculture workers, and the number of physicians per 100,000 people from Taiwan census database [37].

\section{Dengue epidemics in study area}

Dengue fever is a notifiable infectious disease in Taiwan. The dengue surveillance data from the Taiwan Centers for Disease Control (Taiwan CDC) are based on institutional reporting and border surveillance. The confirmed dengue cases reported by the Taiwan CDC are laboratory-positive dengue cases, which indicates a suspected dengue case with anti-dengue IgM seroconversion or single anti-dengue IgM positivity or a case with

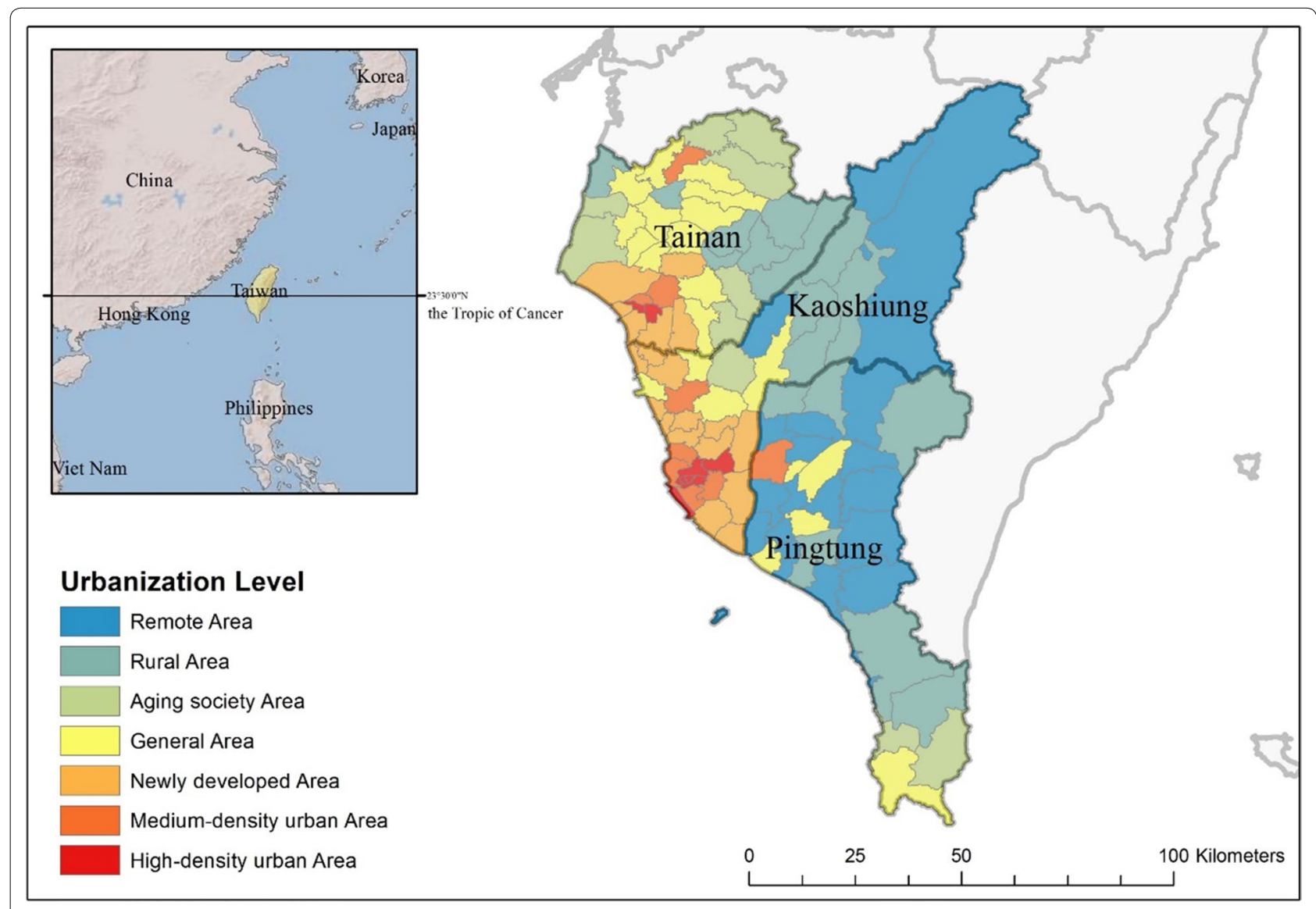

Fig. 1 Urbanization levels in southern Taiwan 
dengue virus identification through RT-PCR [38]. Their residences of cases were also aggregated as counts in townships for public announcement. Figure 2 shows the temporal trend of dengue epidemics from 1998 to 2015 in Taiwan. The figure indicates that, in the last 2 years, the number of confirmed cases reached 15,732 and 43,784, and 233 people dead, respectively, which are the most severe outbreaks over the course of 70 years in Taiwan. Most high-epidemic areas were concentrated in Tainan and Kaohsiung Cities (Fig. 3). Moreover, the southern Taiwan is located in the border of tropical and sub-tropical climatic zones (Fig. 1). Therefore, the dengue epidemics in Taiwan can be regarded as one of dengue sentinel indicators in Southeast and East Asia, which monitors geographic expansion of dengue epidemics to middle or high-latitude countries.

Figure 4 shows the monthly variations of dengue cases in southern Taiwan, 2014 and 2015, and it indicates that there were significant epidemic seasons in these 2 years. We defined the month with the highest dengue cases as the start of epidemic season. Therefore, we categorized the periods of October to December of 2014 and September to December of 2015 as epidemic seasons. We further investigated the association of neighborhood structures and dengue diffusion between pre-epidemic and epidemic seasons in these 2 years.
Table 1 summarizes the population density, number of townships and dengue incidence during the pre-epidemic and epidemic periods in 7 urbanization stratifications, including remote, rural, ageing, general, new-developed, medium-density and high-density areas. It shows that dengue cases were concentrated in medium- and highdensity areas in both the pre-epidemic and epidemic stages. Therefore, human mobility between townships with high urbanization levels could be critical routes of spatial transmission.

\section{Spatial weights and neighborhood structures}

Spatial proximity and human mobility may influence neighborhood diffusion of dengue epidemics. Different definitions of neighborhood structures reflect the effects of spatial interactions. We defined three neighborhood structures, including Queen Contiguity, Distance-threshold weights and matrix of human mobility, for investigating the influence of different types of neighborhood structures on dengue diffusion.

\section{Queen contiguity weights}

The queen contiguity is one of the standard contiguitybased spatial weighting methods in geographic analysis. It determines neighboring units as those that have any point in common, including both common boundaries

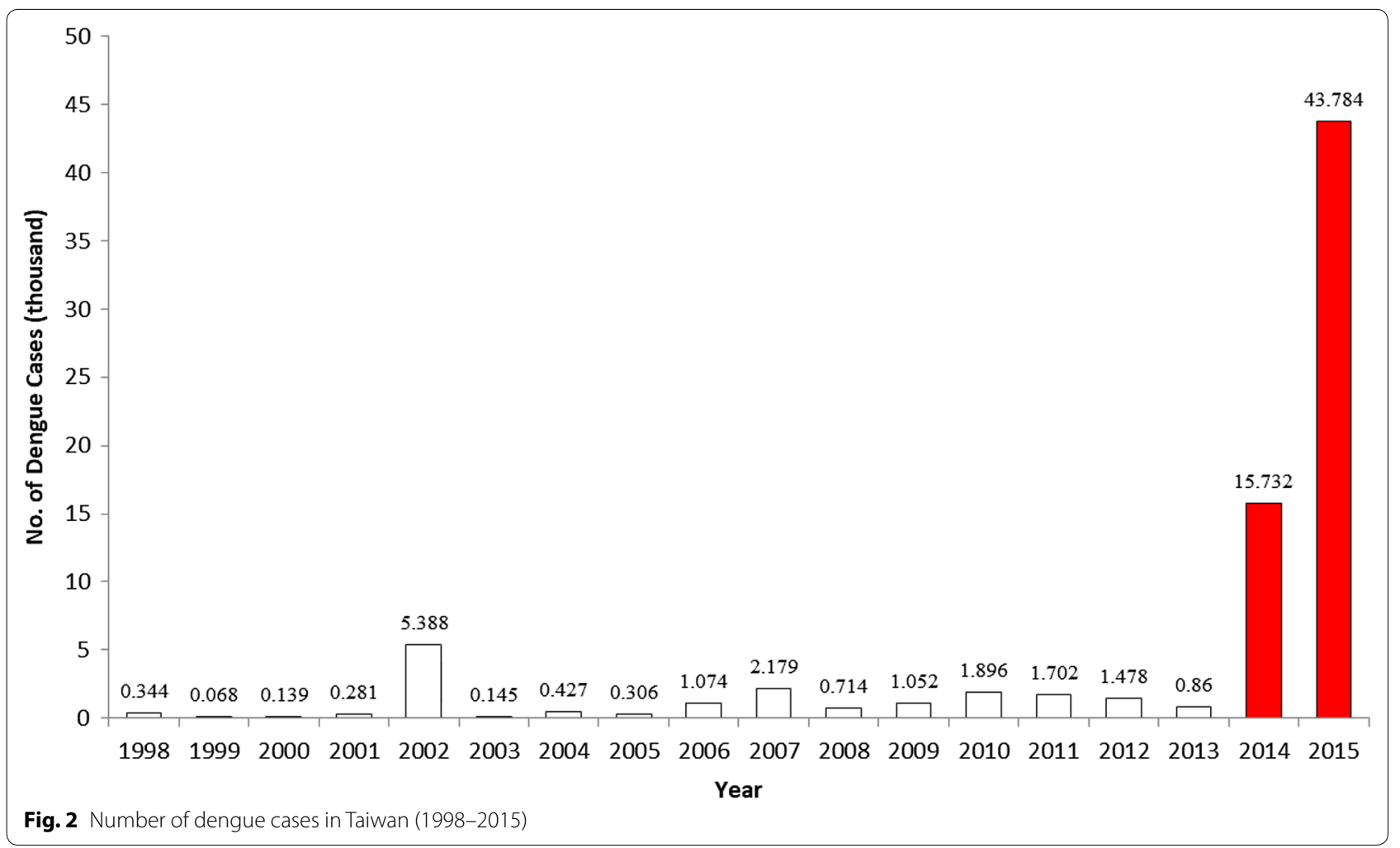



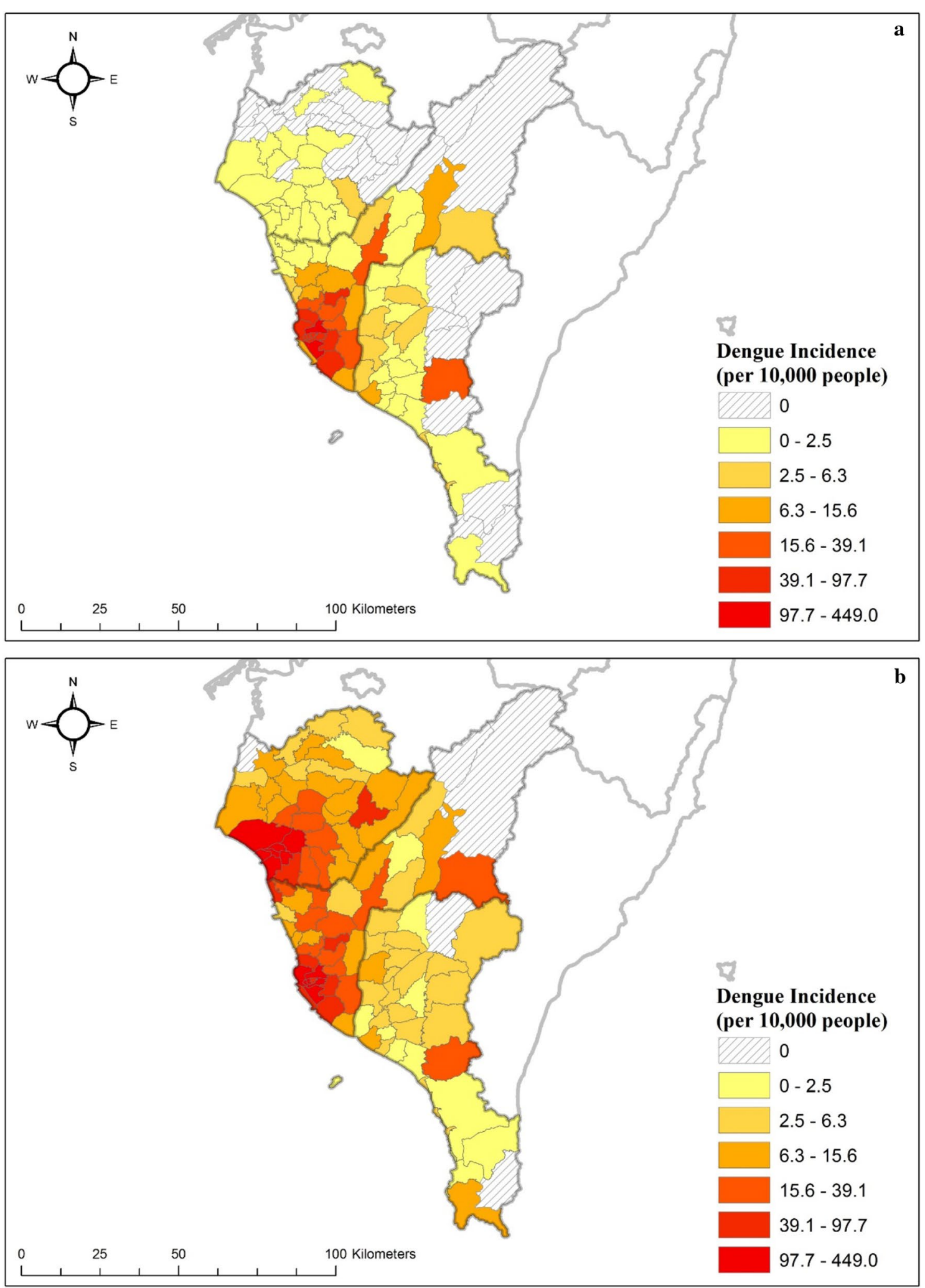

Fig. 3 Spatial distributions of dengue incidence in a 2014 and b 2015 

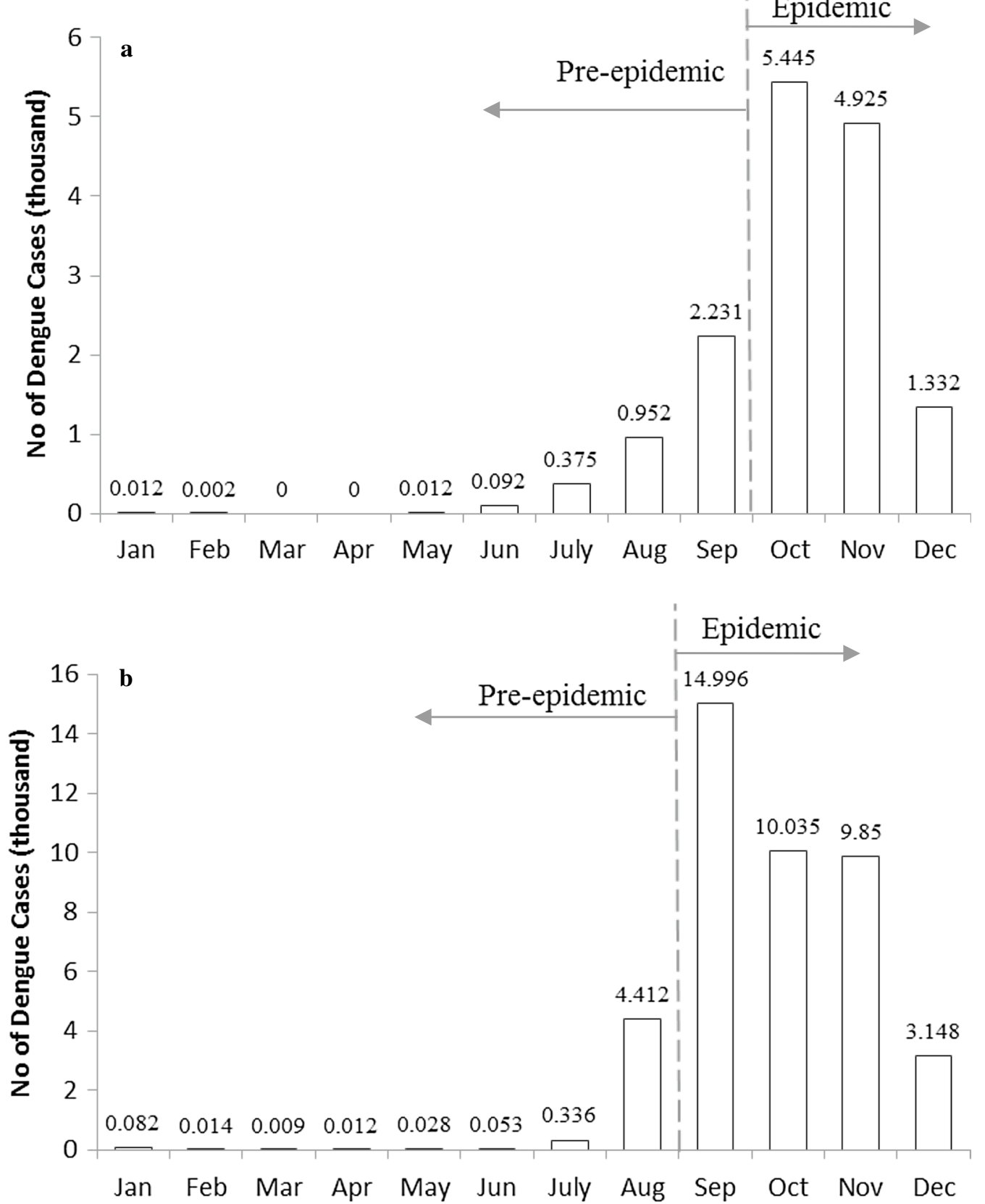

Fig. 4 Monthly number of dengue cases in $\mathbf{a} 2014$ and b 2015. In 2014, the period of Jan.-Sep. is defined as pre-epidemic and Oct.-Dec. as epidemic; in 2015, the period of Jan.-Aug. is defined as pre-epidemic and Sep.-Dec. as epidemic stage

and common corners. The spatial weights in a queen contiguity matrix $\left(\mathrm{W}_{\mathrm{Q}}\right)$ represent townships that share administration boundaries and have higher possibilities of interacting with each other. Based on queen contiguity, each township has an average of 5.14 neighboring townships in our study.

\section{Distance-threshold weights}

The distances among townships could influence the extent of daily mobility. The queen contiguity cannot incorporate the effect of distance on spatial interaction. The extent of daily mobility is measured by the journeys someone takes from home to work and back again. The 
Table 1 Summarized Statistics in urbanization levels

\begin{tabular}{|c|c|c|c|c|}
\hline \multirow[t]{2}{*}{ Township stratification } & \multirow[t]{2}{*}{ Counts of towns } & \multirow[t]{2}{*}{$\begin{array}{l}\text { Avg. population density, } \\
\text { person } / \mathrm{km}^{2}\end{array}$} & \multicolumn{2}{|c|}{$\begin{array}{l}\text { Dengue incidence rate, per } 10,000 \\
\text { people }\end{array}$} \\
\hline & & & Pre-epidemic & Epidemic \\
\hline Remote area & 22 & 132.9 & 1.07 & 5.5 \\
\hline Rural area & 18 & 121.5 & 0.81 & 7.24 \\
\hline Aging society area & 10 & 163.1 & 0.64 & 4.01 \\
\hline General area & 21 & 616.2 & 2.52 & 14.08 \\
\hline Newly developed area & 19 & 1870.8 & 13.56 & 72.5 \\
\hline Medium-density urban area & 9 & 5421.5 & 24.69 & 116.23 \\
\hline High-density urban area & 9 & $10,746.7$ & 24.97 & 196.16 \\
\hline
\end{tabular}

Institute of Transportation identified $20 \mathrm{~km}$ as the average daily journey distance for urban trips [39]. Therefore, we measured distances between centroids of townships for establishing a spatial weight matrix $\left(\mathrm{W}_{\mathrm{D}}\right)$ that defined the townships within $20 \mathrm{~km}$ as the criteria of a neighborhood for modeling spatial interactions. In other words, the distance-threshold weights can reflect spatial interactions between townships within $20 \mathrm{~km}$.

\section{Matrix of human mobility}

The weights of Queen Contiguity and Distance-threshold reflect geometric characteristics of neighborhood structures rather than the patterns of human mobility. In other words, these definitions cannot differentiate the directions and volumes of population flow between urban-tourban and urban-to rural areas. Therefore, we adopted the concept of radiation model proposed by Simini et al. [32] for quantifying spatial interaction between townships (Eq. 1). The radiation model can estimate routine human mobility, which reflects daily commute [32]. Therefore, we used the proportions of commuters from one to another location as the spatial weight matrix for quantifying spatial associations among locations.

$$
T_{i j}=m_{i} \frac{m_{j}}{\left(m_{i}+s_{i j}\right)\left(m_{i}+m_{j}+s_{i j}\right)}
$$

where $T_{i j}$ is the proportion of the commuters in township $\mathrm{i}$ travelling to township $\mathrm{j}$; $\mathrm{mi}$ and $\mathrm{m}_{\mathrm{j}}$ are the populations in townships $i$ and $j$, respectively; and $s_{\mathrm{ij}}$ is the total population in the circle centered at $i$ and touching $j$, excluding the source and destination population as shown Fig. 5a. The population in the circle $\left(\mathrm{s}_{\mathrm{ij}}\right)$ represents attraction (e.g., opportunity of jobs) to mi. If $\mathrm{s}_{\mathrm{ij}}$ is larger, it indicates that the population in the $\mathrm{m}_{\mathrm{i}}$ has more mobility alternatives, which decreases the mobility propensity from $m_{i}$ to $\mathrm{m}_{\mathrm{j}}$. The parameter-free model is validated in various behaviors of human mobility, including journeys with a short travel time, daily commute, and migration [32]. We used the model to estimate the trips for constructing an Original-Destination Matrix W, W(i,j) is the estimated trips from township $i$ to $j$ and the transpose matrix $\mathrm{W}^{\mathrm{T}}(\mathrm{i}, \mathrm{j})$ is then the estimated trips from $\mathrm{j}$ to $\mathrm{i}$. Therefore, we generated a fully connected symmetric matrix $\mathrm{W}_{\mathrm{F}}=\mathrm{W}+\mathrm{W}^{\mathrm{T}}$, which can capture the spatial interactions between townships, and $\mathrm{W}_{\mathrm{F}}$ is the spatial weights we used to measure the human mobility between townships (Fig. 5b). Township population statistical data for the radiation model is from the Department of Household Registration, Ministry of the Interior in Taiwan.

Figure 6 illustrates an example of the neighborhood structures of Fongshan District in Kaohsiung City based on these three criteria. The neighborhood structures in Fig. 6a, b reflect the geometric characteristics of the administration boundary, and the spatial interactions in Fig. $6 \mathrm{c}$ capture the spatial variations of human mobility and characteristics of urbanization.

\section{Statistical analysis}

We used spatial regression modeling for investigating the neighborhood effects on spatial-time diffusion of dengue incidence between a township and its neighboring area. Spatial lag model, one of spatial regression specifications, adds a spatial lag operator to the outcome variable (e.g. disease incidence) for investigating neighboring effects as diffusion process [40, 41]. Therefore, by integrating dengue cases in different periods, in this study, spatial lag model was used for quantitatively measuring dengue diffusion effects in different periods.

We developed three statistical model specifications: Model 1 only measures the neighboring effect of the preepidemic period ( $\mathrm{t} 1$ ), and Model 2 considers the diffusion effect of dengue incidence in neighboring townships during both the pre-epidemic (t1) and epidemic (t2) periods. Comparing these two models can differentiate diffusion 

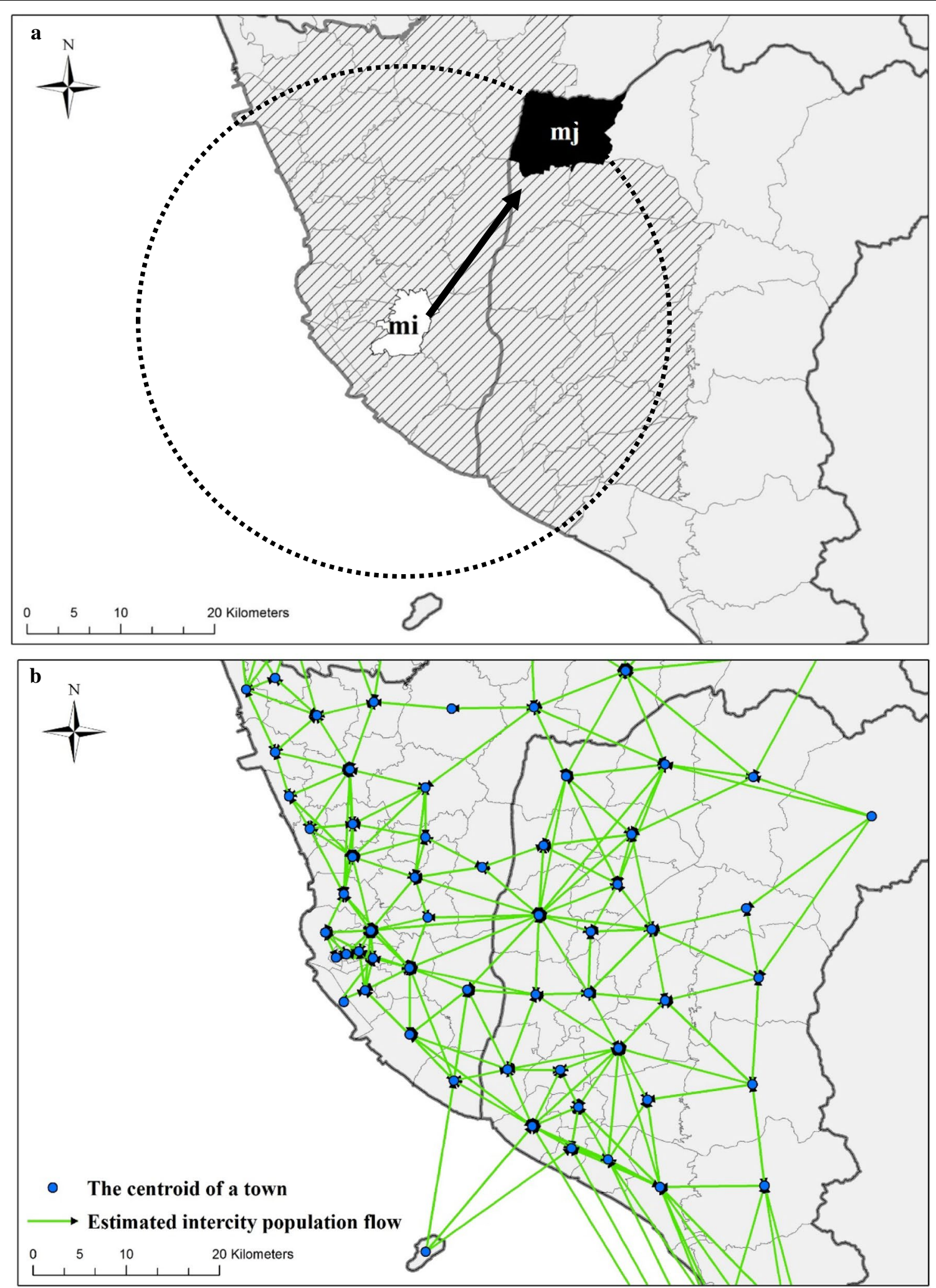

Fig. 5 Illustrations of $\mathbf{a}$ an example of using a radiation mode to estimate population flow from $m_{i}$ (source) to $m_{j}$ (destination), which considers total population in the circle centered at $m_{i}$ and touching $m_{j}$ excluding the source $\left(m_{i}\right)$ and the destination $\left(m_{j}\right)$ population. More population in the circle represent people in the mi have more attractive (e.g. opportunity of jobs), and it decreases mobility propensity from mi to $m_{j j}$ b network connectivity structure of human mobility estimated by a radiation model to represent spatial interaction 

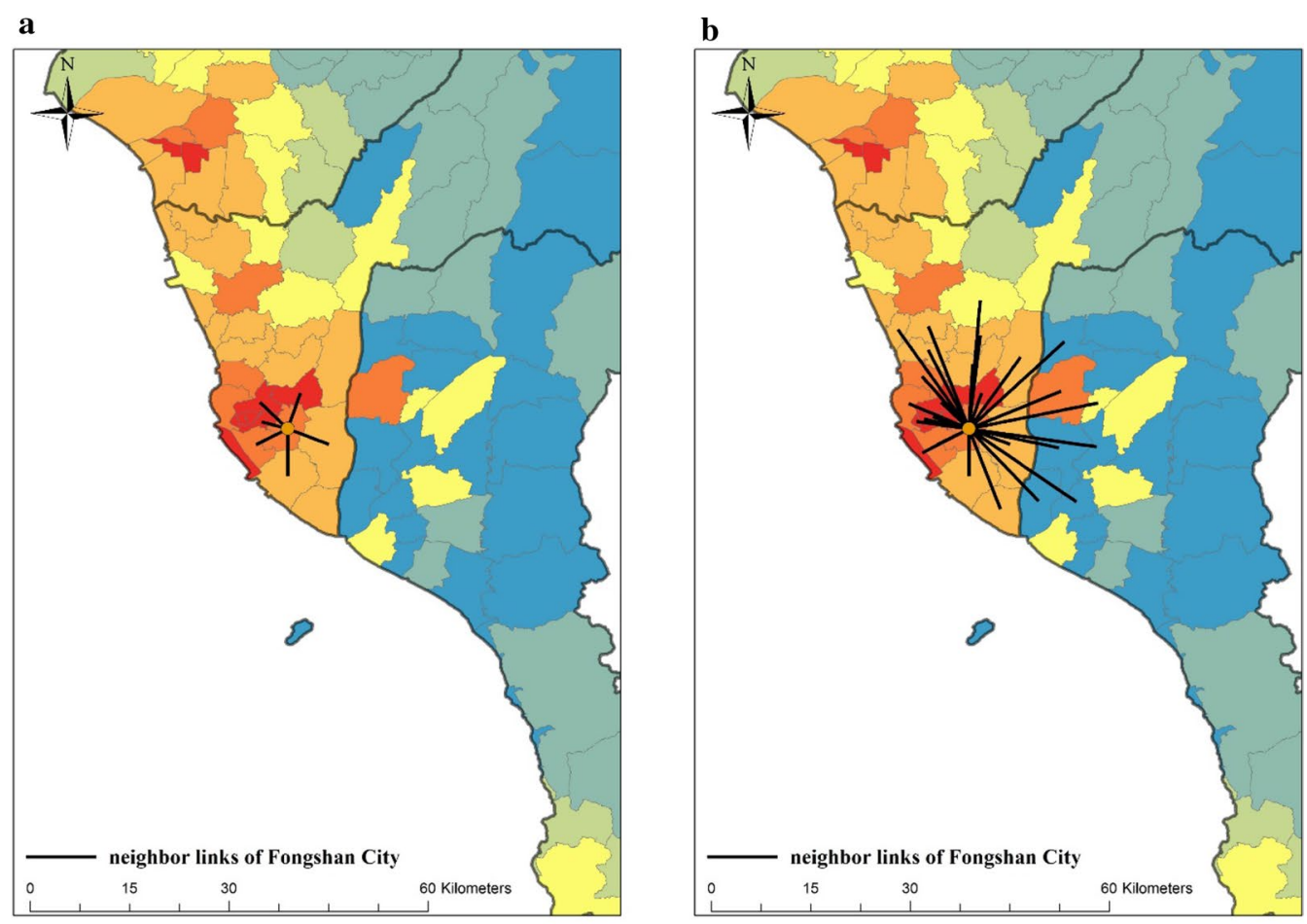

c

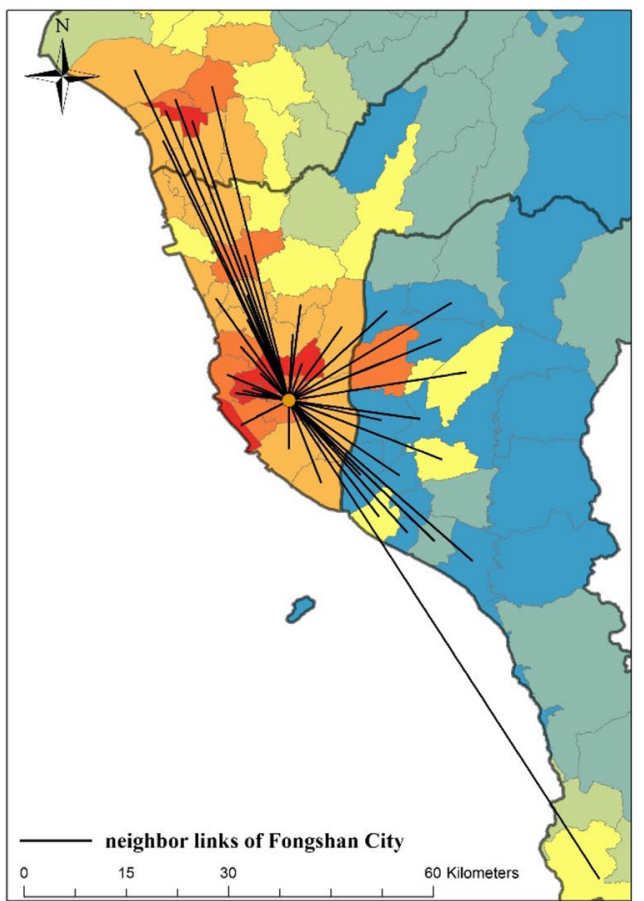

\section{Urbanization Level}

\begin{tabular}{|c|}
\hline Remote Area \\
\hline Rural Area \\
\hline Aging society Area \\
\hline General Area \\
\hline Newly developed Area \\
\hline Medium-density urban Area \\
\hline High-density urban Area \\
\hline
\end{tabular}

Fig. 6 An example of neighborhood structures of Fongshan District of Kaohsiung City based on three definitions: a queen contiguity, $\mathbf{b}$ distance-threshold weights and $\mathbf{c}$ structure of human mobility

effects from the pre-epidemic (t1) and epidemic (t2) periods to investigate the influence of neighborhood structures on epidemic progression. Model 3 considers the second-order neighboring townships of the pre-epidemic period (t1) for quantifying the relatively long-distance diffusion effect during the period. 
Moreover, different settings of neighborhood structures, including Queen Contiguity, distance-threshold weights and structure of human mobility, are compared in each model for each year so that we could systematically understand which neighborhood structure is more appropriate for the discussion on spatial autocorrelation of dengue and the result we found would be more convinced. Models 1 was fitted to data using ordinary least squares (OLS) method and Models 2 and 3 were fitted using maximum likelihood estimation (MLE) with the $R$ package spdep. The Akaike information criterion (AIC) is used as the performance indicator of model fitting. A model with a lower AIC value has a better explanation for dengue diffusion.

The model framework of statistical analysis is shown in Fig. 7. Different colors of the layers represent different-order neighborhood structures. The variables in the first layer (blue) represent a township i; the second layer (green) represent the 1-order neighborhood (immediate neighbors), and the third layer (pink) represent the 2-order neighborhood (distant neighbors). The arrows represents influence relations between these variables. Detailed model specifications are described as follows.

\section{Model 1: pre-epidemic neighborhood effect model}

We used ordinary least squares (OLS) Regression to investigate the diffusion effect of dengue incidence in neighboring townships during the pre-epidemic period, controlling for urbanization levels, as shown in Eq. 2

$$
\mathrm{y}_{t 2}=\sum_{a=1}^{6} \beta_{a} x_{a}+\rho_{t 1} W \mathrm{y}_{t 1}+\varepsilon
$$

where $y_{t 2}$ is the logarithmic dengue incidence of a township during the epidemic period ( $\mathrm{t} 2$ ) and $\mathrm{x}$ is the urbanization level, which is a categorical variable. There are six dummy variables used to capture seven urbanization levels. $\beta_{\mathrm{a}}$ is the marginal effect for one urbanization type (a). W is a spatial weight matrix, including the abovementioned $\mathrm{W}_{\mathrm{Q}}, \mathrm{W}_{\mathrm{D}}$ and $\mathrm{W}_{\mathrm{F}}$, for investigating the neighborhood structures. $W y_{t 1}$ measures dengue incidence in pre-epidemic period (t1) and its coefficient $\rho_{t 1}$ is the marginal neighboring effect during the pre-epidemic period (t1), which can capture diffusion process of dengue epidemics from $t 1$ to $t 2 . \varepsilon$ is the regression residual.

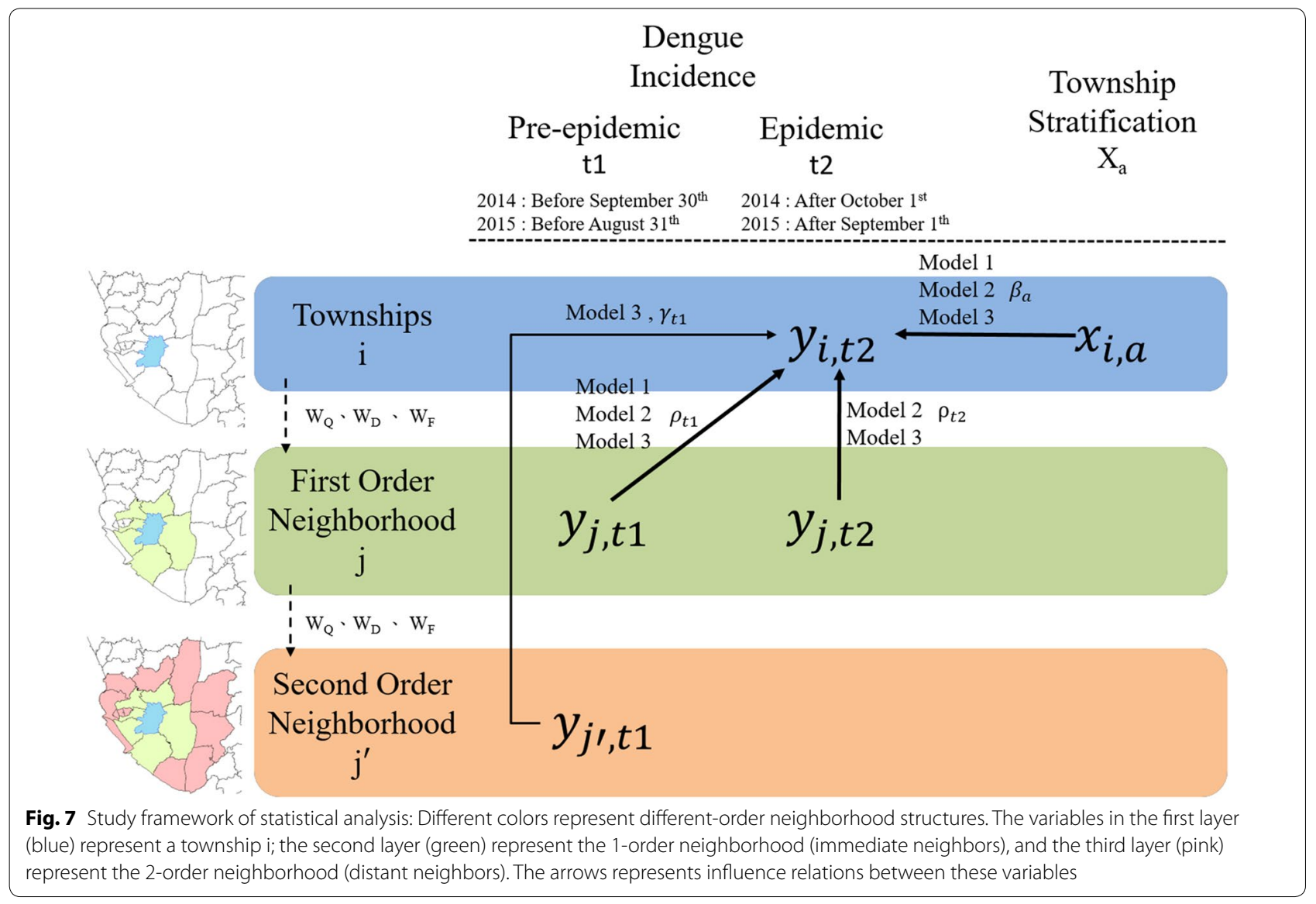




\section{Model 2: current neighborhood effect model}

We used spatial lag models (SLM) to investigate the diffusion effect of dengue incidence in neighboring townships during both the pre-epidemic (t1) and epidemic (t2) periods, controlling for urbanization levels, as shown in Eq. 3.

$$
\mathrm{y}_{t 2}=\rho_{t 2} \mathrm{~W} y_{t 2}+\sum_{a=1}^{6} \beta_{a} x_{a}+\rho_{t 1} W y_{t 1}+\varepsilon
$$

where $\beta_{\mathrm{a}}$ is the marginal effect for one urbanization type (a). W is a spatial weight matrix, including the above-mentioned $\mathrm{W}_{\mathrm{Q}}, \mathrm{W}_{\mathrm{D}}$ and $\mathrm{W}_{\mathrm{F}}$, for investigating the neighborhood structures. Similar as Model $1, \rho_{t 2} \mathrm{~W} y_{t 2}$ measures diffusion effect of dengue incidence in epidemic period $(t 2)$. $\rho_{\mathrm{t} 2}$ is the marginal neighboring effect during the epidemic period (t2), and $\rho_{\mathrm{t} 1}$ is the effect during the pre-epidemic period (t1), and $\varepsilon$ is the regression residual.

\section{Model 3: long distance model}

We used spatial Durbin models (SDM) to investigate the 2-order neighborhood effect in the pre-epidemic period as shown in Eq. 4. The 2-order neighborhood for a township refers to the neighbors of neighboring townships. In other words, the 2-order neighboring effect can capture the effect of relatively long-distance diffusion. Model 3 is the same as Model 2, except for the term $\gamma_{t 1} W\left(W y_{t 1}\right)$, which captures 2-order neighboring townships.

$$
y_{t 2}=\rho_{t 2} \mathrm{~W} y_{t 2}+\sum_{a=1}^{6} \beta_{a} x_{a}+\rho_{t 1} W y_{t 1}+\gamma_{t 1} W\left(W y_{t 1}\right)+\varepsilon
$$

where $\beta_{\mathrm{a}}$ is the marginal effect for one urbanization type (a). W is a spatial weight matrix, including the abovementioned $\mathrm{W}_{\mathrm{Q}}, \mathrm{W}_{\mathrm{D}}$ and $\mathrm{W}_{\mathrm{F}}$, for investigating the neighborhood structures. $\rho_{\mathrm{t} 2}$ is the marginal neighboring effect during the epidemic period ( $\mathrm{t} 2) ; \rho_{\mathrm{t} 1}$ is the effect during the pre-epidemic period ( $\mathrm{t} 1) ; \gamma_{\mathrm{t} 1}$ is the marginal 2-order neighboring effect during the pre-epidemic period $(t)$; and $\varepsilon$ is the regression residual.

\section{Results}

Tables 2 and 3 summarize the effects of different model configurations and epidemic progression on dengue incidence in 2014 and 2015.

Our findings show consistent results for the dengue epidemics of 2014 and 2015.

Table 4 summarized the model-fitting performances (AIC values) for different settings of neighborhood structures and spatial model configurations for dengue diffusion in 2014 and 2015. It shows that Model 3 (long distance model) with structure of human mobility has the lowest AIC value. (Detailed statistical results for all of the models can be found in Additional file 1: Tables S1S4). This finding indicates that population mobility as the neighborhood structure can better explain the relatively

Table 2 Model results of $\mathbf{2 0 1 4}$ dengue epidemic using the matrix of population mobility as spatial weights

\begin{tabular}{llll}
\hline Independent variables & Model 1 & Model 2 & Model 3 \\
\hline Intercept & $1.527(0.79)$ & $1.149(0.69)$ & $3.039^{* * *}(0.75)$ \\
Spatial-lag of dengue incidence & & & \\
1st order neighbors in pre-epidemic period $\rho_{t 1}$ & $1.107^{* * *}(0.08)$ & $0.671^{* * *}(0.11)$ & $-0.002(0.17)$ \\
1st order neighbors in epidemic period $\rho_{t 2}$ & - & $-428^{* * *}(0.09)$ & $0.306^{* *}(0.10)$ \\
2nd order neighbors in pre-epidemic period $\gamma_{t 1}$ & - & & $1.001^{* * *}(0.20)$ \\
Urbanization levels ${ }^{\mathrm{a}}$ & & $-0.099(0.21)$ & $0.015(0.198)$ \\
Rural area $\beta_{1}$ & $-0.229(0.24)$ & $0.569^{*}(0.27)$ & $0.384(0.25)$ \\
Aging society area $\beta_{2}$ & $0.641^{*}(0.31)$ & $0.26(0.21)$ & $0.465^{*}(0.20)$ \\
General area $\beta_{3}$ & $0.236(0.24)$ & $0.647^{* *}(0.22)$ & $0.771^{* * *}(0.20)$ \\
Newly developed area $\beta_{4}$ & $0.964^{* * *}(0.24)$ & $0.595^{*}(0.27)$ & $0.639^{*}(0.24)$ \\
Medium-density urban area $\beta_{5}$ & $0.791^{*}(0.30)$ & $0.313(0.29)$ & $0.456(0.27)$ \\
High-density urban area $\beta_{6}$ & $0.521(0.33)$ & & 226.01 \\
Performance of model fitting & & 247.11 & - \\
AlC & 259.54 & - & \\
R-square & 0.72 & &
\end{tabular}

The value in parentheses is standard error

*p value $<0.05 ;$ **p value $<0.01$; ***p value $<0.001$

a "Remote area" as reference category 
Table 3 Model results of $\mathbf{2 0 1 5}$ dengue epidemic using the matrix of population mobility as spatial weights

\begin{tabular}{llll}
\hline Independent variables & Model 1 & Model 2 & Model 3 \\
\hline Intercept & $-3.08^{* * *}(0.90)$ & $-1.56(0.81)$ & $-0.53(0.92)$ \\
Spatial-lag of dengue incidence & & & \\
1st order neighbors in pre-epidemic period $\rho t 1$ & $0.50^{* * *}(0.09)$ & $0.27^{*}(0.08)$ & $-0.007(0.16)$ \\
1st order neighbors in epidemic period $\rho t 2$ & - & $-47^{* * *}(0.08)$ & $0.467^{* * *}(0.08)$ \\
2nd order neighbors in pre-epidemic period $y t 1$ & - & & $0.40^{*}(0.19)$ \\
Urbanization levels & & $-0.10(0.21)$ & $-0.14(0.20)$ \\
Rural area $\beta_{1}$ & $-0.11(0.25)$ & $-0.45(0.25)$ & $-0.53(0.25)$ \\
Aging society area $\beta_{2}$ & $-0.37(0.30)$ & $0.40(0.21)$ & $0.33(0.20)$ \\
General area $\beta_{3}$ & $0.42(0.24)$ & $0.77^{* *}(0.24)$ & $0.66^{*}(0.24)$ \\
Newly developed area $\beta_{4}$ & $1.34^{* * *}(0.26)$ & $1.31^{* * *}(0.29)$ & $1.21^{* * *}(0.29)$ \\
Medium-density urban area $\beta_{5}$ & $1.94^{* * *}(0.32)$ & $1.44^{* * *}(0.33)$ & $1.36^{* * *}(0.32)$ \\
High-density urban area $\beta_{6}$ & $2.47^{* * *}(0.33)$ & & 237.17 \\
Performance of model fitting & & -23.52 & - \\
AlC & 259.25 & - & \\
R-square & 0.708 & & \\
\hline
\end{tabular}

The value in parentheses is standard error

*p value $<0.05$; **p value $<0.01 ;{ }^{* * *}$ p value $<0.001$

a "Remote area" as reference category

long-distance (2nd-order) and immediate (1st-order) neighboring dengue diffusion in different periods.

Regarding urbanization levels in the models with population mobility structures, in Tables 2 and 3, Model 3 shows areas that are newly developed, medium-density and high-density are associated with significantly higher dengue incidence relative to remote areas. Interestingly, the results of Model 3 also indicate that the dengue incidence during the epidemic period is significantly associated with 1st-order neighbors during the epidemic period (t2) and 2nd-order neighbors which are relatively distant townships compare with 1st-order neighbors during the pre-epidemic period (t1) since the coefficient of $\rho_{t 2}$ and $\gamma_{t 1}$ in Model 3 are significant. Our results show the spatial-temporal hierarchy of dengue diffusion: the dengue incidence in a township would be impacted by immediate neighbors during pre-epidemic and epidemic periods, and also with more distant neighbors (based on mobility) in pre-epidemic periods.

\section{Discussion}

Recent literature has indicated that one of the major driving forces of geographic expansion of dengue is human mobility on different scales, including within-city, intercity in a region, and international travels $[13,15,16]$. To quantify the effects of mobility on the spatial diffusion of dengue epidemics, previous literature replaced spatial mobility structures with geometric relationships in spatial regression models [4, 25]. However, these geometric relationships cannot fully reflect realistic spatial interactions $[27,28]$. On the other hand, large-scale intercity individual mobility routes are difficult to collect, track, and access in most countries. Therefore, our study proposed a framework for model configuration to profile intercity dengue diffusion. First, we used a radiation model to construct the structure of human mobility as neighborhood weight in the spatial regression model. Second, we categorized the pre-epidemic and epidemic periods for investigating the time-lag effect on dengue

Table 4 AIC values for different settings of neighborhood structures and spatial model configurations for dengue diffusion

\begin{tabular}{|c|c|c|c|c|c|c|}
\hline \multirow[t]{2}{*}{ Neighborhood structures } & \multicolumn{3}{|l|}{2014} & \multicolumn{3}{|l|}{2015} \\
\hline & Model 1 & Model 2 & Model 3 & Model 1 & Model 2 & Model 3 \\
\hline Queen contiguity & 328.4 & 319.7 & 320.6 & 278.0 & 278.1 & 275.7 \\
\hline Distance-threshold weights & 256.0 & 254.3 & 255.0 & 270.7 & 268.0 & 269.9 \\
\hline Structure of human mobility & 259.5 & 247.1 & 226.0 & 259.3 & 239.5 & 237.2 \\
\hline
\end{tabular}

Model 1: pre-epidemic neighborhood effect model, Model 2: current neighborhood effect model, and Model 3: long distance model 
diffusion. Lastly, we incorporated 2nd-order and 1storder neighboring structures in our model to quantify relatively long-distance and immediate diffusion effects. Compared with conventional model configuration in spatial regression analysis, our proposed radiation model specification demonstrates better model fitting performance in both 2014 and 2015, which indicates that the structure of human mobility has better explanatory power in dengue diffusion than geometric relationships. The model results in the 2014 and 2015 dengue epidemics have consistent findings, indicating that intercity mobility and urbanization could be driving forces of large-scale epidemic expansion of dengue [12, 14, 17, 19].

Our findings show that highly-urbanized areas are positively associated with dengue incidence in 2014 and 2015 , which is consistent with the literature, such as in $[3,4,42]$. In southern Taiwan, household plant plotting in townhouse buildings and small-area flooding water in yards after an extensive rainfall provide appropriate habitats for dengue vector mosquitoes in urbanized areas. Meanwhile, areas with high population densities could help mosquitoes bite people more easily [4]. On the other hand, female Aedes aegypti, a major dengue vector mosquito, are most active during the daytime, which means that there is a high frequency of biting by $A$. aegypti for people gathering in public places, which increases the risk of dengue outbreak [43-45].

Regarding geographic expansion of epidemics, our study identifies a significant neighboring diffusion effect on dengue epidemics. The dengue incidence in a township would be impacted by neighboring townships during either the pre-epidemic or epidemic period. This finding implies that the potential sources of diffusion for the township might be its neighboring townships with high dengue incidence. The result reflects the structure of human mobility as spatial interactions causing epidemic expansion [4, 25]. Moreover, the 2nd-order neighboring structure also has a significant effect on the township during the pre-epidemic period, which reflects the relatively long-distance diffusion effect. In other words, our results demonstrate a "ripple" process of dengue diffusion, which means that the immediate (first-order) neighboring effect occurs in the initial epidemic wave and that the wider geographic expansion occurs in a later epidemic wave, which is affected by the distant (secondorder) neighboring effect.

Cliff et al. [46] categorized spatial diffusion patterns into three major types. Contagion results from direct contact for spreading. Relocation describes diffusion source shifts to another distant location. Hierarchy refers to transmission through an ordered sequence of settlements rather than following a distance-based neighborhood structure. Numerous studies have interpreted the epidemiological implications of these diffusion patterns [47-49]. In most cases, the structure of epidemic diffusion is often a mixture of these patterns $[13,49,50]$, and relocation and hierarchical patterns cause long-distance dispersion [51]. Our proposed model has profiled possible mechanisms for these patterns. Conventional geometric relationships, such as contiguity-based and distance-threshold weighting schemes, are based on a distance-decayed structure, which could capture the characteristics of contagious diffusion. However, geometric relationships do not reflect topographic variability and long-distance interactions due to transportation. A radiation model considered in our study captures the structure of intercity interactions, reflecting patterns of human mobility. For example, in Fig. 6c, population flows from Fongshan city (high-density urbanized areas) are not only to neighboring townships, but there are also flows to other distant high-density cities in Tainan. This means that our model can capture realistic urban-tourban interactions partially, which could cause relocation and hierarchical diffusion. Brockmann and Helbing [23] also proposed a concept of "effective distance," which replaces conventional geographic distance with the matrix of passenger flux through air traffic between cites for predicting global disease arrival times. In summary, large-scale geographic expansion of epidemic propagation is difficult to explain only by the geometric structure of administration boundaries or geographic distances of centroids between cities. Population mobility or passenger flows could more reasonably capture the structure of spatial interactions and long-distance diffusion patterns. In other words, hub cities could play a role as a "bridge" for large-scale transmission and make townships connecting to hub cities more vulnerable to dengue epidemics.

The study has some limitations. First, the mobility structure was estimated by a parameter-free radiation model, which only considers the population size of a city rather than the empirical or surveyed mobility data. Although the estimated mobility structure captures urban-to-urban interactions, we did not consider the detailed mobility behaviors of individuals or even dengue patients, such as choices of transportation modes or purposes of the trip. Further investigation on more detailed intercity mobility structure is warranted. Second, in addition to human mobility, dengue diffusion is a complex process in terms of the spatial-temporal variability in mosquito density, effectiveness of control measures, pathogen activity and host immunity [43, 52]. However, most of these factors are not available for intercity-scale studies. It is necessary to develop reliable sampling schemes for collecting these data in further investigations. Thirdly, we only used the most severe epidemics 
in Taiwan as case study. Although the findings are consistent in both years, it does not imply the findings still valid in other years. It would be worth to incorporate long-term longitudinal epidemic data for investigating the influence of human mobility on dengue diffusion. Our findings may suggest that, in severe epidemic years, human mobility plays a significant role in intercity dengue diffusion. Finally, the spatial heterogeneity of intercity diffusion effect should also be considered in further investigation. For example, a geographic weighted regression (GWR) can be further used to differentiate the effect of human mobility on dengue incidence in each township. Spatially-varying relationships of neighboring effects on dengue incidence could provide the heath authority for implementing better adopt specific control and prevention strategies to specific areas.

\section{Conclusions}

The study proposed a study framework for investigating relatively long-distance and immediate neighboring diffusion effects on epidemic propagation and clarified the role of intercity-scale human mobility structure and urbanization levels as driving forces in large-scale dengue transmission. Our findings suggest that the intercity mobility structure reflects urban-to-urban interactions, which causes a mixture of relocation and hierarchical diffusion patterns for large-scale dengue epidemics in southern Taiwan. This can be identified as a "ripple" process wherein an immediate neighboring effect occurs in the first stage and wider geographic expansion occurs in a later stage, which is influenced by the distant neighboring diffusion effect.

\section{Additional file}

Additional file 1. Detailed statistical results for all of the models.

\begin{abstract}
Abbreviations
AIC: Akaike information criterion; AR5: The Fifth Assessment Report; CDC: Centers of Disease Control; CDRS: call detail records; IPCC: Intergovernmental Panel on Climate Change; GPS: Global Positioning System; GWR: geographically weighted regression; OLS: ordinary least squares; SDM: spatial Durbin model; SLM: spatial lag model.
\end{abstract}

\section{Authors' contributions}

THW conceived the experiments. THW and CSH conducted the experiments and analyzed the data. THW, CSH and MCH interpreted the results and wrote the paper. All authors read and approved the final manuscript.

\section{Author details}

${ }^{1}$ Department of Geography, National Taiwan University, No. 1, Sec. 4, Roosevelt Road, Taipei City 10617, Taiwan. ${ }^{2}$ Department of Bioenvironmental Systems Engineering, National Taiwan University, No. 1, Sec. 4, Roosevelt Road, Taipei City 10617, Taiwan.

\section{Acknowledgements}

None.

\section{Competing interests}

The authors declare that they have no competing interests.

\section{Availability of data and materials}

The datasets generated and/or analyzed during the current study are available from the corresponding author on reason request.

\section{Consent for publication \\ Not applicable.}

Ethics approval and consent to participate

Not applicable.

\section{Funding}

The research was supported by Grants from the Ministry of Science and Technology in Taiwan (MOST 105-2627-M-002-018 and MOST 105-2410-H-002150-MY3). The authors also acknowledge the financial support provided by the Infectious Diseases Research and Education Center, the Ministry of Health and Welfare (MOHW) and National Taiwan University (NTU) and NTU Research Center for Future Earth (NTU-107L9010). The funders had no role in the study design, data collection and analysis, or manuscript preparation.

\section{Publisher's Note}

Springer Nature remains neutral with regard to jurisdictional claims in published maps and institutional affiliations.

Received: 5 December 2017 Accepted: 26 April 2018

Published online: 03 May 2018

\section{References}

1. Galvani AP, May RM. Epidemiology: dimensions of superspreading. Nature. 2005;438(7066):293.

2. Bhatt $S$, Gething PW, Brady OJ, Messina JP, Farlow AW, Moyes CL, Drake JM, Brownstein JS, Hoen AG, Sankoh O. The global distribution and burden of dengue. Nature. 2013;496(7446):504

3. Qi X, Wang Y, Li Y, Meng Y, Chen Q, Ma J, Gao GF. The effects of socioeconomic and environmental factors on the incidence of dengue fever in the Pearl River Delta, China, 2013. PLoS Negl Trop Dis. 2015;9(10):e0004159.

4. Wu P-C, Lay J-G, Guo H-R, Lin C-Y, Lung S-C, Su H-J. Higher temperature and urbanization affect the spatial patterns of dengue fever transmission in subtropical Taiwan. Sci Total Environ. 2009;407(7):2224-33.

5. Åström C, Rocklöv J, Hales S, Béguin A, Louis V, Sauerborn R. Potential distribution of dengue fever under scenarios of climate change and economic development. EcoHealth. 2012;9(4):448-54.

6. Bouzid M, Colón-González FJ, Lung T, Lake IR, Hunter PR. Climate change and the emergence of vector-borne diseases in Europe: case study of dengue fever. BMC Public Health. 2014;14(1):781.

7. Hales S, De Wet N, Maindonald J, Woodward A. Potential effect of population and climate changes on global distribution of dengue fever: an empirical model. Lancet. 2002;360(9336):830-4.

8. Kutsuna S, Kato Y, Moi ML, Kotaki A, Ota M, Shinohara K, Kobayashi T, Yamamoto K, Fujiya Y, Mawatari M. Autochthonous dengue fever, Tokyo, Japan, 2014. Emerg Infect Dis. 2015;21(3):517.

9. Patz JA, Martens W, Focks DA, Jetten TH. Dengue fever epidemic potential as projected by general circulation models of global climate change. Environ Health Perspect. 1998;106(3):147.

10. Stocker TF, Qin D, Plattner G-K, Tignor M, Allen SK, Boschung J, Nauels A, Xia Y, Bex V, Midgley PM: Climate change 2013: the physical science basis. In: Contribution of working group I to the fifth assessment report of the intergovernmental panel on climate change. Cambridge University Press, Cambridge; 2013.

11. Sutherst RW. Global change and human vulnerability to vector-borne diseases. Clin Microbiol Rev. 2004;17(1):136-73. 
12. Thai KT, Anders KL. The role of climate variability and change in the transmission dynamics and geographic distribution of dengue. Exp Biol Med. 2011;236(8):944-54.

13. Kan C-C, Lee P-F, Wen T-H, Chao D-Y, Wu M-H, Lin NH, Huang SY-J, Shang C-S, Fan I-C, Shu P-Y. Two clustering diffusion patterns identified from the 2001-2003 dengue epidemic, Kaohsiung, Taiwan. Am J Trop Med Hyg. 2008;79(3):344-52.

14. Itoda I, Masuda G, Suganuma A, Imamura A, Ajisawa A, Yamada K-I, Yabe S, Takasaki T, Kurane I, Totsuka K. Clinical features of 62 imported cases of dengue fever in Japan. Am J Trop Med Hyg. 2006;75(3):470-4.

15. Stoddard ST, Forshey BM, Morrison AC, Paz-Soldan VA, Vazquez-Prokopec GM, Astete H, Reiner RC, Vilcarromero S, Elder JP, Halsey ES. House-tohouse human movement drives dengue virus transmission. Proc Natl Acad Sci. 2013;110(3):994-9.

16. Wesolowski A, Qureshi T, Boni MF, Sundsøy PR, Johansson MA, Rasheed SB, Engø-Monsen K, Buckee CO. Impact of human mobility on the emergence of dengue epidemics in Pakistan. Proc Natl Acad Sci. 2015;112(38):11887-92.

17. Wesolowski A, Eagle N, Tatem AJ, Smith DL, Noor AM, Snow RW, Buckee CO. Quantifying the impact of human mobility on malaria. Science. 2012;338(6104):267-70

18. Shang C-S, Fang C-T, Liu C-M, Wen T-H, Tsai K-H, King C-C. The role of imported cases and favorable meteorological conditions in the onset of dengue epidemics. PLoS Negl Trop Dis. 2010;4(8):e775.

19. Wen $\mathrm{T}-\mathrm{H}$, Lin M-H, Fang C-T. Population movement and vector-borne disease transmission: differentiating spatial-temporal diffusion patterns of commuting and noncommuting dengue cases. Ann Assoc Am Geogr. 2012;102(5):1026-37.

20. Vazquez-Prokopec GM, Stoddard ST, Paz-Soldan V, Morrison AC, Elder JP, Kochel TJ, Scott TW, Kitron U. Usefulness of commercially available GPS data-loggers for tracking human movement and exposure to dengue virus. Int J Health Geogr. 2009;8(1):68.

21. de Castro Medeiros LC, Castilho CAR, Braga C, de Souza WV, Regis L, Monteiro AMV. Modeling the dynamic transmission of dengue fever: investigating disease persistence. PLOS Negl Trop Dis. 2011;5(1):e942.

22. Barmak DH, Dorso CO, Otero M, Solari HG. Dengue epidemics and human mobility. Phys Rev E. 2011;84(1):011901.

23. Brockmann D, Helbing D. The hidden geometry of complex, networkdriven contagion phenomena. Science. 2013;342(6164):1337-42.

24. Gardner LM, Sarkar S. Risk of dengue spread from the Philippines through international air travel. Transp Res Rec J Transp Res Board. 2015;2501:25-30.

25. Tipayamongkholgul M, Lisakulruk S. Socio-geographical factors in vulnerability to dengue in Thai villages: a spatial regression analysis. Geospat Health. 2011;5(2):191-8.

26. Almeida ASD, Medronho RDA, Valencia LIO. Spatial analysis of dengue and the socioeconomic context of the city of Rio de Janeiro (Southeastern Brazil). Rev Saude Publica. 2009;43(4):666-73.

27. Chi Y, Jhou M, Hsieh Y. Spatial analysis of foreign brides in Taiwan. J Popul Stud. 2009;38(3):67-113.

28. Rincke J. A commuting-based refinement of the contiguity matrix for spatial models, and an application to local police expenditures. Reg Sci Urban Econ. 2010:40(5):324-30.

29. Tizzoni M, Bajardi P, Decuyper A, King GKK, Schneider CM, Blondel V, Smoreda Z, González MC, Colizza V. On the use of human mobility proxies for modeling epidemics. PLoS Comput Biol. 2014;10(7):e1003716.

30. Vazquez-Prokopec GM, Bisanzio D, Stoddard ST, Paz-Soldan V, Morrison AC, Elder JP, Ramirez-Paredes J, Halsey ES, Kochel TJ, Scott TW. Using GPS technology to quantify human mobility, dynamic contacts and infectious disease dynamics in a resource-poor urban environment. PLOS ONE. 2013;8(4):e58802.
31. de Dios OrtÃozar J, Willumsen LG. Modelling transport. New York: Wiley; 2011.

32. Simini F, González MC, Maritan A, Barabási A-L. A universal model for mobility and migration patterns. Nature. 2012;484(7392):96.

33. Corbel V, Nosten F, Thanispong K, Luxemburger C, Kongmee M, Chareonviriyaphap T. Challenges and prospects for dengue and malaria control in Thailand, Southeast Asia. Trends Parasitol. 2013;29(12):623-33.

34. World Health Organization (WHO) Regional Office for South-East Asia Comprehensive guidelines for prevention and control of dengue and dengue haemorrhagic fever: Revised and expanded edition. New Delhi: WHO; 2011.

35. Shepard DS, Undurraga EA, Halasa YA. Economic and disease burden of dengue in Southeast Asia. PLoS Negl Trop Dis. 2013;7(2):e2055.

36. Undurraga EA, Halasa YA, Shepard DS. Use of expansion factors to estimate the burden of dengue in Southeast Asia: a systematic analysis. PLoS Negl Trop Dis. 2013;7(2):e2056.

37. Liu C-Y, Hung Y, Chuang Y, Chen Y, Weng W, Liu J, Liang K. Incorporating development stratification of Taiwan townships into sampling design of large scale health interview survey. J Health Manag. 2006;4(1):1-22.

38. King C-C, Wu Y-C, Chao D-Y, Lin T-H, Chow L, Wang H-T, Ku C-C, Kao C-L, Chien L-J, Chang H-J. Major epidemics of dengue in Taiwan in 1981-2000: related to intensive virus activities in Asia. Dengue Bull. 2000;24:1-10.

39. Lin K, Su C, Chang C, Leu H, Chang S, Yang Y. The demand model of intercity transportation systems under national sustainable development in Taiwan (2/4). Taipei: Ministry of Transportation and Communications, Institute of Transportation; 2006.

40. Abreu M, de Groot HL, Florax RJ. Spatial patterns of technology diffusion: an empirical analysis using TFP, Discussion Paper No. 04079/3. Amsterdam-Rotterdam: Tinbergen Institute; 2004.

41. Ng I-C, Wen T-H, Wang J-Y, Fang C-T. Spatial dependency of tuberculosis incidence in Taiwan. PLoS ONE. 2012;7(11):e50740.

42. Vallée J, Dubot-Pérès A, Ounaphom P, Sayavong C, Bryant JE, Gonzalez JP. Spatial distribution and risk factors of dengue and Japanese encephalitis virus infection in urban settings: the case of Vientiane, Lao PDR. Trop Med Int Health. 2009:14(9):1134-42.

43. Chadee D, Shivnauth B, Rawlins S, Chen A. Climate, mosquito indices and the epidemiology of dengue fever in Trinidad (2002-2004). Ann Trop Med Parasitol. 2007;101(1):69-77.

44. Gubler DJ. Dengue and dengue hemorrhagic fever. Clin Microbiol Rev. 1998;11(3):480-96

45. Wen $\mathrm{T}-\mathrm{H}$, Lin $\mathrm{M}-\mathrm{H}$, Teng $\mathrm{H}-\mathrm{J}$, Chang $\mathrm{N}-\mathrm{T}$. Incorporating the human-Aedes mosquito interactions into measuring the spatial risk of urban dengue fever. Appl Geogr. 2015;62:256-66

46. Cliff AD. Spatial diffusion: an historical geography of epidemics in an island community, vol. 14. Cambridge: CUP Archive; 1981.

47. Cohen J, Tita G. Diffusion in homicide: exploring a general method for detecting spatial diffusion processes. J Quant Criminol. 1999;15(4):451-93.

48. Meade MS, Emch M. Medical geography. New York: Guilford Press; 2010.

49. Hsueh Y-H, Lee J, Beltz L. Spatio-temporal patterns of dengue fever cases in Kaoshiung City, Taiwan, 2003-2008. Appl Geogr. 2012;34:587-94.

50. Ruan S, Wang W, Levin SA. The effect of global travel on the spread of SARS. Math Biosci Eng. 2006:3(1):205.

51. Yang Y, Atkinson PM. Individual space-time activity-based model: a model for the simulation of airborne infectious-disease transmission by activity-bundle simulation. Environ Plan. 2008;35(1):80-99.

52. Sanchez L, Vanlerberghe V, Alfonso L, del Carmen Marquetti M, Guzman MG, Bisset J, Van Der Stuyft P. Aedes aegypti larval indices and risk for dengue epidemics. Emerg Infect Dis. 2006;12(5):800. 